\title{
BMJ Open Study protocol for the TRUSt trial: a pragmatic randomised controlled trial comparing the standard of care with a transitional pain service for patients at risk of chronic postsurgical pain undergoing surgery
}

Manouk Admiraal (1) , Henning Hermanns, Jeroen Hermanides, Carin G.C.L. Wensing, Soe L. Meinsma, Hans C. H. Wartenberg, Martin V. H. Rutten, Vivian M. C. Ward - van der Stam, Markus W. Hollmann

To cite: Admiraal M, Hermanns $\mathrm{H}$, Hermanides $\mathrm{J}$, et al. Study protocol for the TRUSt trial: a pragmatic randomised controlled trial comparing the standard of care with a transitional pain service for patients at risk of chronic postsurgical pain undergoing surgery. BMJ Open 2021;11:e049676. doi:10.1136/ bmjopen-2021-049676

- Prepublication history and additional supplemental material for this paper are available online. To view these files, please visit the journal online. (http://dx.doi.org/10.1136/ bmjopen-2021-049676).

Received 29 January 2021 Accepted 28 July 2021

Check for updates

(c) Author(s) (or their employer(s)) 2021. Re-use permitted under CC BY-NC. No commercial re-use. See rights and permissions. Published by BMJ.

Department of Anesthesiology, Amsterdam UMC - Locatie AMC, Amsterdam, The Netherlands

Correspondence to Dr Jeroen Hermanides; j.hermanides@amsterdamumc. $\mathrm{nl}$

\section{ABSTRACT}

Introduction Patients with either surgery-related or patient-related risk factors are at an increased risk of acute and chronic postsurgical pain (CPSP) and longterm opioid use. To improve recovery, prevent CPSP and decrease opioid use, we need to identify these patients before surgery and provide a multidisciplinary pain management strategy throughout hospital admission and follow-up in the postdischarge period. We hypothesise that a multidisciplinary transitional pain service (TPS) improves quality of recovery and reduce the incidence of CPSP and opioid consumption.

Methods and analysis We aim to investigate the effectiveness of implementation of a TPS for patients at risk of developing CPSP. The trial design is a pragmatic, open-label, randomised controlled trial (RCT). After stratification for sex, patients are randomly assigned to the TPS or standard of care (SOC) group. Our primary outcome is the quality of recovery, measured at the morning of the third postoperative day, employing the quality of recovery (Q0R)-15 questionnaire. Secondary outcomes are the incidence of CPSP, opioid consumption and patient-reported outcome measures at 3 and 6 months postoperatively. We need to enrol 176 patients to detect a minimal clinical important difference of 8 points on the QoR-15 score.

Ethics and dissemination Ethics approval was obtained by the accredited medical research ethics committee of the Academic Medical Center in Amsterdam (2020_211) on 15 0ctober 2020. Protocol version 3.2 was approved on 25 January 2020 . The trial is registered with the Netherlands Trial Register, NL9115. The results will be disseminated by open access publication in a peerreviewed journal.

\section{Trial registration number NL9115}

\section{INTRODUCTION}

Globally, over 320 million people undergo major surgery each year, of which
Strengths and limitations of the study

- This study is the first randomised controlled trial (RCT) comparing a transitional pain service (TPS) with standard of care for patients at risk of chronic postsurgical pain (CPSP).

- This is a pragmatic RCT and will therefore provide real-world evidence on the use of TPS.

- The primary outcome is a patient-reported outcome measure, which takes into account all aspects of quality of recovery, including pain, physical comfort and independence, psychological support and emotional state.

- TPS team and patients cannot be blinded due to the nature of the study.

- The standard of care group might also benefit from implementation of TPS due to an increased awareness for CPSP among healthcare givers (Hawthorne effect).

approximately $10 \%$ will develop chronic postsurgical pain (CPSP). ${ }^{12}$ CPSP is often underdiagnosed and poorly managed, thereby placing a major burden on patient's daily life resulting in significant health problems. In addition, patients with CPSP often take high dosages of opioids due to inappropriate opiate prescribing. ${ }^{3}$ Major risk factors for CPSP include chronic pain before surgery, preoperative opioid exposure and the intensity of acute postoperative pain. ${ }^{4}$ Key is to identify these patients before surgery and provide multidisciplinary pain management throughout hospital admission, a so called transitional pain service (TPS). Studies on the effectiveness of TPS are scarce, but some studies do support further research 
into the implementation of TPS. Tiippana et al retrospectively collected data from medical records and determined whether referral of surgical patients to an Acute Pain Service Out-Patient Clinic (APS-OPC) was effective in reducing opioid use in the immediate postoperative period at home. ${ }^{5}$ At discharge, $54 \%$ of the patients were using weak opioids and $32 \%$ strong opioids. This was reduced to $20 \%$ and $6 \%$ after implementation of the APSOPC. Also, the Toronto General Hospital launched the first prospective study on TPS in 2014. ${ }^{6}$ Patients at high risk of developing CPSP were referred to TPS to manage pain, maintain musculoskeletal function and to lower opioid consumption. Six months postoperatively, opioidnaive and opioid-experienced patients reduced opioid use by $69 \%$ and $44 \%$, respectively. Thus, these studies justify further prospective randomised studies on the effectiveness of TPS.

The aim of our study is therefore to investigate the effectiveness of implementation of a multidisciplinary TPS team for patients at risk of developing CPSP, as measured by the quality of recovery, the incidence of CPSP and the postoperative opioid consumption. We hypothesise that a multidisciplinary TPS improves quality of recovery and reduce the incidence of CPSP and opioid consumption.

\section{METHODS}

For the content of this protocol we used the Standard Protocol Items: Recommendations for Interventional Trials (SPIRIT) and the SPIRIT patient-reported outcome (PRO) extension guidelines. Besides that, this trial implements the Consolidated Standards of Reporting Trials (CONSORT) guidelines. ${ }^{7-10}$ The trial is registered with the Netherlands Trial Register, NL9115 (online supplemental appendix 1).

\section{Study design}

The TRUSt study is a randomised single-centre, parallel grouping, two-armed, superiority trial with a 1:1 allocation ratio. The study is being conducted in an urban tertiary referral teaching hospital in the Netherlands. Approximately 12000 patients undergo surgery in the Amsterdam UMC, location AMC, each year.

\section{Eligibility criteria}

Patients are eligible for inclusion if they fulfil the following criteria:

- 18 years and older, Dutch-speaking and reading

- Willing and able to provide informed consent

- Undergoing a surgical procedure with an increased risk of CPSP (amputation, spinal surgery, thoracotomy, mastectomy, herniotomy, hysterectomy and arthroplasty). ${ }^{11}$

Or, any surgery and meeting one or more of the following criteria:

- Diagnosed with chronic pain, defined according to the International Classification of Diseases (ICD)-11 as 'an unpleasant sensory and emotional experience associated with actual or potential tissue damage, or described in terms of such damage. Chronic pain is pain that persists or recurs for longer than 3 months'. ${ }^{12}$

- Patients with pain device implants, such as intrathecal pain pump, spinal cord stimulation or peripheral nerve stimulator.

- Chronic opioid use, defined as consumption of more than 20 morphine milligram equivalents (MME) per day for more than 3 months in the last 3 months (online supplemental appendix 2).

- Allergy to opioids

- The usage of pain medication as methadone, buprenorphine, anticonvulsants, antidepressants or medicinal cannabis for chronic pain for more than 3 months in the last 3 months.

- Psychosocial comorbidities like anxiety, depression, pain catastrophising if documented in the electronic medical record.

\section{Exclusion criteria}

- Patients who undergo emergency surgery are excluded to ensure sufficient time for the informed consent process.

- Patients undergoing implementation of pain device implants, such as intrathecal pain pump, spinal cord stimulators or peripheral nerve stimulator.

- Patients who undergo surgery that most likely leads to prolonged sedation and for that reason cannot fill in the QoR-15 questionnaire at day 3 postoperative.

\section{Recruitment strategies}

Patients are recruited at the anaesthesiology outpatient preoperative evaluation (OPE) clinic, due to COVID-19 mostly by phone. Trained study personnel informs the patient about the study. If the patient gives permission, a member of the research team calls and informs the patient about the purpose, nature and duration of this study. Besides that the risks and benefits are fully explained. Due to logistics because of SARS-CoV-2, randomisation is performed after verbal informed consent is provided. Patients sign on the day of admission, before surgery and are blinded for randomisation until they have signed the consent form (online supplemental appendix 3).

\section{Study outline}

Patient enrollment started on 18 January 2021 and the study is expected to end in December 2022. After informed consent is provided, patient characteristics are recorded (box 1) and the patient is randomised. Study duration, including follow-up, is 6 months. During the study, patients will complete different questionnaires, at six different time points (figure 1).

\section{Randomisation and blinding}

Patients are randomly assigned to TPS or standard of care (SOC) in a 1:1 ratio. Treatment assignments are performed centrally using a computer-generated random schedule in permuted blocks of four, six or eight with stratification for sex. 


\section{Box 1 The patient characteristics}

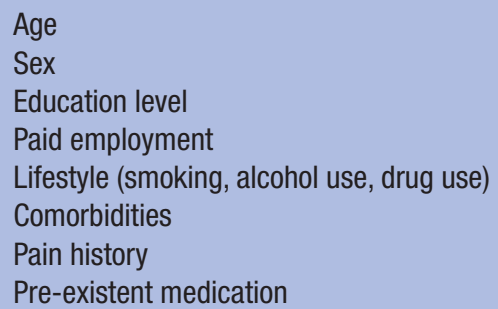

The study is not blinded for patients or study staff. The outcome assessor will be blinded to treatment allocation by receiving the raw dataset coded and without having access to information about the allocation. Figure 2 is the CONSORT flow diagram and includes estimates for eligible, screened, enrolled and analysed patients.

\section{Study treatment}

Control (SOC) group

Patients in the SOC group (figure 3) will receive SOC. This includes preassessment at the OPE clinic, during which medical screening is performed, the perioperative anaesthetic and analgesic strategy is discussed and perioperative pain management is planned. For perioperative analgesia, the practice guidelines for Acute Pain management in the perioperative setting are adhered to. ${ }^{13}$ After surgery, on the ward, nurses, supervised by surgeons, hold a great deal of responsibility for pain management. In addition, the consultative service of the acute pain team (APS) can be requested. The APS is indicated for patients in pain after recent surgery or trauma. Commonly used modalities for pain treatment by the APS include epidural analgesia, peripheral nerve catheter or patient controlled analgesia. A specialised APS nurse (supervised by an anaesthesist) visits each patient once or twice per day. The APS is available 24 hours a day, 7 days a week. Postsurgical opioids are prescribed by the surgeon. From that moment on, both the surgeon and the general practitioner could approve a series of repeat prescriptions. In the Netherlands, $99 \%$ of the population is insured for health expenses. The health insurance consists of care provided by a general practitioner, who they can refer themselves to when needed.

\section{Intervention (TPS) group}

For patients randomised to the intervention group (figure 4), the TPS focuses on patient-centred continuity of care. This starts preoperatively and continues until 6 months after discharge. The TPS team is led by three anaesthesist who are specialised in acute and/or chronic pain management and consists of nurse practitioners, a pain psychologist, a physiotherapist, a social worker and a $\mathrm{PhD}$ researcher. After preoperative screening, patients receive a folder with a brief and simple explanation about pain and patient empowerment to facilitate coping with their condition. ${ }^{14}$ Preoperatively, the patient is discussed in the TPS team according to a standard format (online supplemental appendix 4). Here, an individualised perioperative pharmacological and/or interventional pain management strategy is agreed on. The multimodal pain approach according to the guidelines produced by the American Society of Anesthesiologists is leading. ${ }^{13}$ During this multidisciplinary meeting, the need for referral to a pain psychologist, physiotherapist or social worker will be discussed and initiated when deemed necessary. Afterwards, one of the TPS members calls the patient to explain the perioperative analgesic strategy. Then, to enhance patient autonomy, decisions about care and treatment are made collaboratively between the patient and the healthcare professional (shared decision-making).

After discharge, follow-up occurs every 2 weeks for 2 months and then every month for the remaining 4 months, or till adequate pain control is achieved and opioids are weaned off completely. The definition of follow-up is a telephone call or an appointment at the outpatient clinic. At this follow-up consultation, progress of the patient and the pain treatment plan are evaluated. When possible, opioids are tapered or discontinued. In the postdischarge period, the patient's general practitioner is called by a member of the TPS team and provided with information on the further pain treatment strategy. In this postdischarge period, additional consultation of the TPS team is possible if the treatment goals are not achieved. If the patient develops CPSP within 6 months after surgery or did not wean off opioids completely, we refer the patient to our chronic pain team.

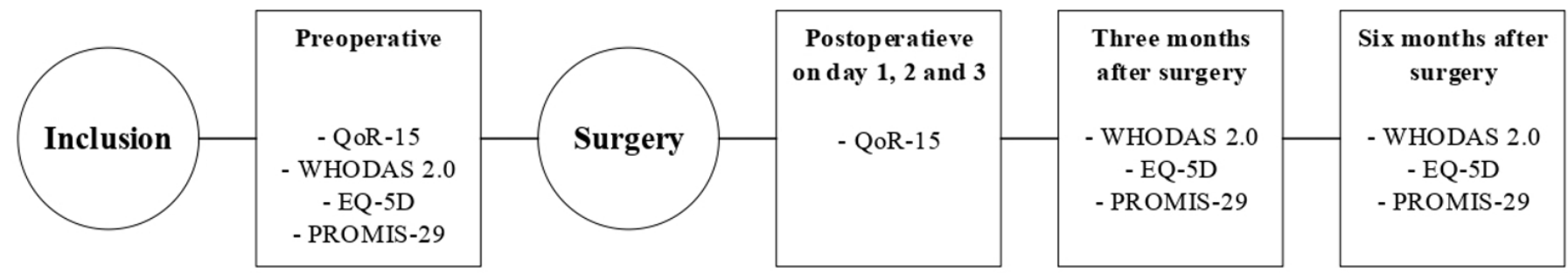

Figure 1 The study assessment flow diagram. EQ-5D, EuroQol-5D; PROMIS, patient-reported outcomes measurement information system; QoR-15, quality of recovery-15 WHODAS 2.0, The WHO Disability Assessment Schedule .0. 


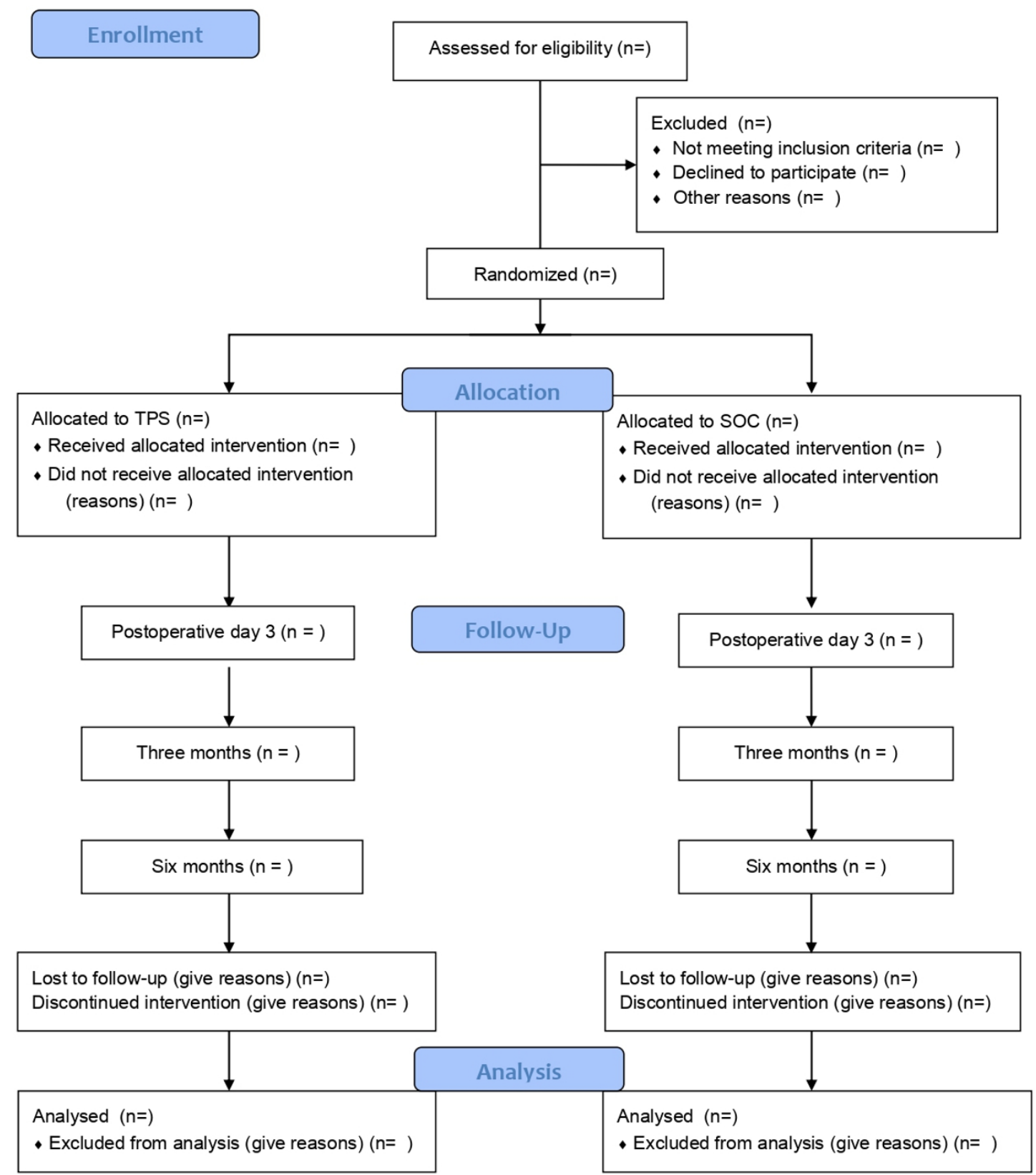

Figure 2 Consolidated Standards of Reporting Trials flow diagram estimating patient screening, enrollment and response rate. SOC, standard of care; TPS, transitional pain service.

\section{Outcomes}

\section{Primary outcome}

Our primary outcome is the quality of recovery, using the quality of recovery (QoR)-15 questionnaire, measured at the morning of the third postoperative day. ${ }^{15}$ The transition from acute to chronic pain is a very complex, not fully elucidated process. However, in patients undergoing surgery, CPSP typically begins as acute pain after surgery, that often is difficult to manage. We hope that by the implementation of a TPS, pain in this primary phase is better controlled and thus results in less transition phase to CPSP. The QoR-15 questionnaire evaluates the patients' initial recovery post-surgery.

For constructs such as pain, comfort or emotional state, the patient's perception is the only source of information and therefore patient-reported outcome measures (PROMs) should be considered the gold-standard evaluation. A well validated patient outcome questionnaire is an objective evaluation that quantifies the patients' pain, recovery as perceived by the patient. ${ }^{16}$ The QoR-15 questionnaire is a validated, reliable and objective PROM as described in several studies. ${ }^{17}$ By taking the questionnaire on the morning of the third postoperative day, we effectively assess the second postoperative day.

\section{Secondary outcomes}

A TPS is not only targeting this acute postoperative phase and therefore we evaluate long-term outcomes in this study as well. Secondary outcomes include postoperative long-term follow-up data: 


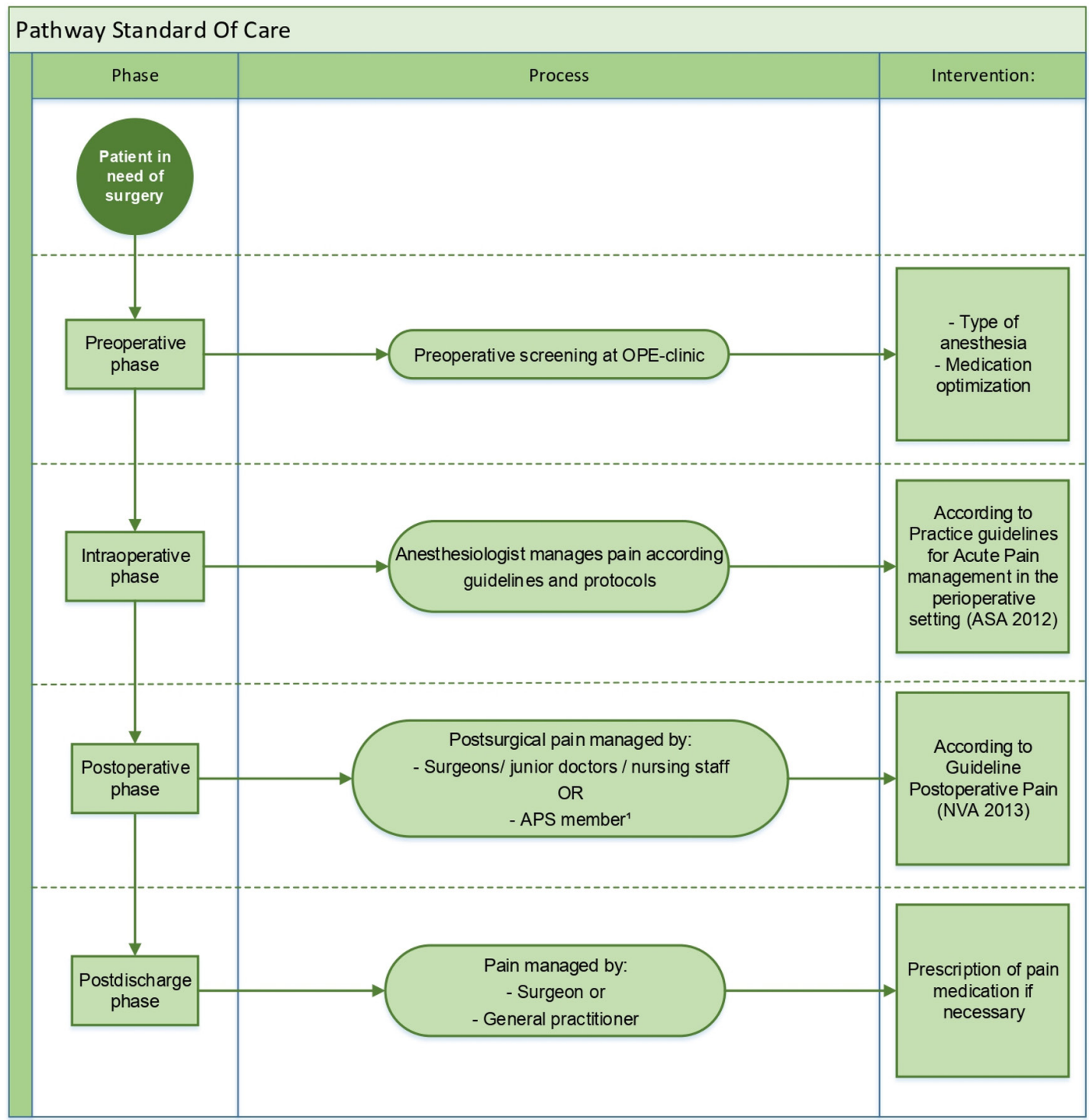

Figure 3 Perioperative pathway for patients allocated to standard of care. 'The APS team is nurse based and anaesthetist supervised. A clinical pain nurse visits each patient on the APS service at least once a day, mostly when pain treatment modalities like intravenous or epidural patient-controlled analgesia, with or without peripheral nerve catheter, are used. The team is in-house 24 hours a day, 7 days a week. when pain medication is switched to oral medication only, the patient is usually discharged from services of the APS. APS, acute pain team; OPE, outpatient preoperative evaluation.

1. CPSP diagnosis (after 3 and 6 months) defined according to the International Association for the Study of Pain (IASP). ${ }^{11}$

2. Opioid consumption (preoperative, postoperative day 3 , after 3 and 6 months): calculated as MME per day.

3. Patient-reported health outcome measurements:

- The WHO Disability Assessment Schedule (WHODAS) 2.0, 12-items: brief assessments that cover six domains of functioning including cognition, mobility, self-care, getting along, life activities and participation. ${ }^{18}$ Scoring has three steps: summing of recorded item scores within each domain, summing of all six domain scores and lastly converting the summary score into a metric ranging from 0 to 100 (where $0=$ no disability; $100=$ full disability) $\cdot{ }^{19}$ We will analyse the difference across groups at baseline and after
3 and 6 months postoperative. We will also analyse a change in the score over time for each group. A change in score of $5 \%$ or more after surgery is consistent with a clinically important change in disability. ${ }^{20}$

- EuroQol-5D-5 level version (EQ-5D-5L): reflecting generic health status: a 5-item summary measure of overall health status. The descriptive system comprises the dimensions: mobility, self-care, usual activities, pain/discomfort and anxiety/depression. ${ }^{21}$ We will summarise the EQ-5D-5L health state by an index value which reflects how good or bad a health state is according to the preferences of the general population of a country/region. ${ }^{22} \mathrm{~A}$ value set is established that represent the views of the Dutch population. ${ }^{23}$ At a minimum, we will analyse the change 


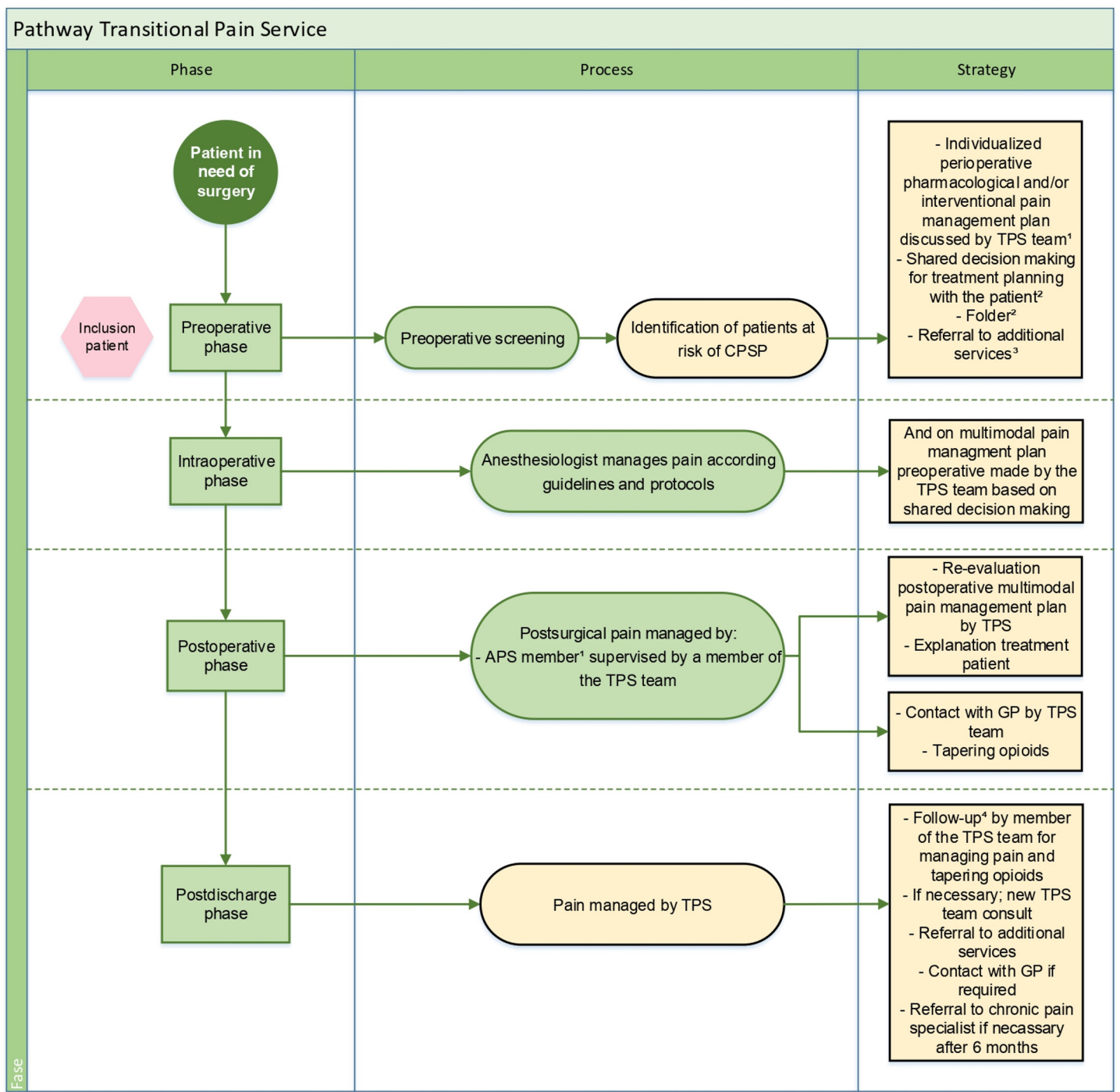

Figure 4 Perioperative pathway for patients allocated to TPS. 1The TPS team consists of three anaesthetists who are specialised in acute and/or chronic pain, pain nurse practitioners, a psychologist, a physiotherapist, a social worker and a PhD student. ${ }^{2}$ Non-pharmacological interventions include an information folder regarding pain and empowerment. Shared decisionmaking about care and treatment to promote patient autonomy during the study. ${ }^{3}$ The TPS team can refer the patient to a psychologist, a social worker or a physiotherapist. ${ }^{4}$ Follow-up after discharge occurs every 2 weeks with a telephone call until his/her pain is under control or medication is weaned off completely. APS, acute pain team; CPSP, chronic postsurgical pain; GP, general practitioner; TPS, transitional pain service.

in index over time within groups (preoperative to 3 and 6 months postoperatively) and between groups. The dimension pain/discomfort will be analysed separately as well.

- Patient-reported outcomes measurement information system (PROMIS)-29: a generic health-related quality of life survey, assesses each of the seven PROMIS domains (anxiety; physical function; pain interference; fatigue; sleep disturbance an disability to participate in social roles an activities), with four questions. The questions are ranked on a 5-point Likert scale. There is also one 11-point rating scale for pain intensity. ${ }^{24}$ Norm-based scores have been calculated for each domain, so that a score of 50 represents the mean of the reference population with a SD of 10 . At a minimum, we will analyse the change in index over time within groups (preoperative to three and 6 months postoperatively) and between groups. The dimension pain/ discomfort will be analysed separately as well.

- QoR-15 comparing changes in time (baseline, day one, two and three postoperatively) within groups. The QoR-15 scores range from 0 (extremely poor) to 150 (excellent quality of recovery). Interventions that result in a change of 8.0 reflect a clinically minimally important difference. ${ }^{25}$

4. Satisfaction staff of the implementation of a TPS, rated on a 5-point Likert scale from 1 (extremely dissatisfied) to 5 (extremely satisfied). ${ }^{26}$

5 . The frequency the perioperative plan (like type of anaesthetic), changed after evaluation of the patient by the TPS team, instead of the earlier discussed method during preoperative assessment. 
Other measurements

- Intraoperative data: type of anaesthesia, doses of opioids, duration of surgery, duration of recovery room stay, and so on.

- Postoperative data: length of hospital stay, method of pain control, dose of opioids and so on.

- Long-term follow-up data: number of contacts with TPS, number of referrals, and so on.

\section{Sample size and drop-out}

Using nQuery Advisor V.8.5.1, sample size is driven by the analysis for superiority of TPS compared with standard care employing the QoR-15 questionnaire score. Assuming a SD of 18 points on postoperative day 3 and being able to detect a QoR-15 score difference of at least 8 (based on the minimal clinically important difference and SD found by Myles et $a l^{25}$ ), randomisation ratio 1:1, a total sample size of 160 patients is required ( 80 patients per group) to detect this difference with a statistical power of $80 \%$ and a significance level of (alpha) 0.05 .

Patients can leave the study at any time for any reason if they wish to do so without any consequences. Patients will not be replaced in case of withdrawal. To account for a possible drop-out rate of $10 \%$, we will include 88 patients per arm, thus a total of 176 .

\section{Protocol deviation}

Protocol deviations or violations could occur in this study and will be reported. An example of a protocol deviation is a follow-up visit at a slightly different time frame than required by protocol, for example, because of the participant's schedule. Furthermore, an anaesthesia technique provided diverging from the one agreed on at the OPE. Besides, a patient allocated to the SOC group who is in severe pain could be discussed in the TPS team because of Good Clinical Practice. Patients with protocol deviations will be included in the intention-to-treat analysis. All protocol violations, except cancelled surgery, will be included in the intention-to-treat analysis but will lead to exclusion from analysis per protocol. When a patient's surgery is cancelled the patient will be excluded from all analysis. There are no safety risks associated with protocol violations in this trial.

\section{Statistical analysis}

Final data will be screened for typos, missing values, outliers and distribution. All data analyses will be carried out according to a pre-established analyses plan. We are planning for complete case analyses and multiple imputations for missing data.

Baseline characteristics, as mentioned in box 1, will be summarised with the use of the appropriate descriptive statistics.

\section{Primary outcome analysis}

All randomised patients will be analysed as the primary population for the analysis according the intention-totreat principle. As mentioned above, cancelled surgery is the only protocol violation that will lead to exclusion from analysis. The primary outcome, the between-group difference in QoR-15 scores will be analysed. Because of the small interval between the intervention and the primary endpoint, we do not expect a significant amount of missing data on the QoR-15 survey. However, patients who are sedated or experiencing a delirium and patients who are discharged before day 3, could cause missing data. Therefore, we will compare responders (patients who returned a completely filled-in QoR-15 questionnaire) and non-responders for differences in patient characteristics, perioperative surgical and anaesthetic factors, to examine non-response bias on age, sex and item response.

Depending on the distribution of the data, we will test the raw between group difference using an unpaired t-test or Mann-Whitney U test. Statistical uncertainties will be quantified with two-sided $95 \%$ CIs. A two-sided p value $<0.05$ will be considered statistically significant. Because of our randomization stratification for sex, we will additionally report effects adjusted for sex.

As part of our secondary analyses, we will perform a per-protocol analysis including all randomised patients completing the whole study period on the between-group difference in QoR-15 scores as described above.

\section{Secondary outcome analysis}

Between-group difference in the proportion of patients having the diagnosis CPSP after 3 and 6 months will be compared using the $\chi^{2}$ test. We expect the change in opioid consumption to be bimodal distributed, some patients will not change their opioid consumption while others will reduce their consumption completely. The between-group difference in change in opioid consumption (MMEs) (postoperative day 3, after 3 and 6 months) will be compared using a generalised linear-mixed model, with treatment as fixed effect and preoperative opioid consumption, time and the interaction between treatment and time as covariates and subject as random factor.

Only if time, or interaction between treatment and time differs significantly between groups, we will perform post hoc analysis. We will use the Benjamini-Hochberg procedure to correct for multiple testing. ${ }^{27}$ Non-normal distribution is expected in WHODAS 2.0, EQ-5D-5L, PROMIS-29 scores and therefore we will analyse the between-group differences at one point using a MannWitney $U$ test and a generalised mixed model. We will use a generalised mixed model to correct for time and to test multiple measurements at the same time. For missing item scores, multiple imputation will be applied. After the study period, staff satisfaction will be measured on implementation of a TPS, using a Mann-Whitney $U$ test and the proportion of perioperative plan changes after evaluation of the TPS team, will be compared using a $\chi^{2}$ test. Finally, an exploratory analyses will be performed by studying differences in treatment effect in subgroups other than sex; different risk factors of CPSP, baseline characteristics and on perioperative treatment. For the exploratory analyses, correction for multiple testing will be applied 
using the Benjamini-Hochberg method. R studio (Affero General Public License V3) will be used for the analyses.

\section{Data collection and management}

Paper and online surveys will be used to collect PROMs. Only Dutch-translated surveys will be used. A automatic reminder will be sent through mail after 3 days (as an exception the QoR-15 survey at day 3 postoperatively, this reminder will be sent after 1 day). If the patient did not complete the survey after 6 days, a manual survey invite will be sent and in case of no response after another 3 days a phone call will be made to the patient who did not complete the survey.

For collecting long-term data at 3 and 6 months, the researcher calls the patients and evaluates if symptoms of CPSP develop and gathers data on the amount of pain medication. At this phone call, the researcher will also remind the patient on the survey Specify PRO data collection and management strategies for minimising avoidable missing data.

The data of each patient will be recorded on an individual electronic case report form (eCRF) using Castor EDC (Ciwit BV the Netherlands, V.1.5, a GCP compliant database). Data will be coded using a unique numerical code. The key to this code is only available to the research team and is stored in the trial master file (TMF) in accordance with the European Union regulation act (General Data Protection Regulation) and GCP. All patient data will be kept confidential. The correctness of entries will be verified for $15 \%$ of the data, by a second investigator. All recorded data, including CRFs, TMF, investigator site file and Informed Consent Form (ICFs) will be stored for 15 years after completion of the study. Study data will always be stored securely, in a locked cabinet or on password-secured computers, only accessible for study team members.

\section{Monitoring}

The study will be monitored, based on a low-risk study design, by a monitor from the Clinical Monitoring Center at the Amsterdam UMC. This is a qualified, independent team that is put in place to monitor according to the monitor plan. The principal investigator and all investigators will permit and facilitate study-related monitoring or regulatory inspection by providing direct access to study files and source data/documents. After each monitoring visit, a site report will be issued by the monitor to the principal investigator and a copy will be provided to the local investigators.

Due to the minimal risk nature of the study, there will be no external data and safety monitoring board.

\section{ETHICS AND DISSEMINATION}

Ethics approval (2020_211) was obtained on 15 October 2020, in the Netherlands at the Medical Research Ethics Committee location Academic Medical Center (Amsterdam, The Netherlands). The trial will be conducted in compliance with this study protocol, the Declaration of Helsinki and GCP. Protocol amendments will be subjected to the Medical Ethics Committee for approval and thereafter communicated to all investigators an trial registries (online supplemental appendix 5 ). There are no publication disclosures.

Contributors MWH, JH, HH, CW, SLM, MR, HCHW, VMCW and MA were involved in conception and trial design. SLM, HCHW, MR, VMCW helped with implementation. MA and SLM will be responsible for the PRO content of the trial. JH, HH and MA were involved in drafting the article. MWH, SLM, HCHW, MR and CW were involved in critical revision of the article. All the authors contributed to refinement of the study protocol and final approval of the article.

Funding The authors have not declared a specific grant for this research from any funding agency in the public, commercial or not-for-profit sectors.

Competing interests None declared.

Patient consent for publication Not required.

Provenance and peer review Not commissioned; externally peer reviewed.

Supplemental material This content has been supplied by the author(s). It has not been vetted by BMJ Publishing Group Limited (BMJ) and may not have been peer-reviewed. Any opinions or recommendations discussed are solely those of the author(s) and are not endorsed by BMJ. BMJ disclaims all liability and responsibility arising from any reliance placed on the content. Where the content includes any translated material, BMJ does not warrant the accuracy and reliability of the translations (including but not limited to local regulations, clinical guidelines, terminology, drug names and drug dosages), and is not responsible for any error and/or omissions arising from translation and adaptation or otherwise.

Open access This is an open access article distributed in accordance with the Creative Commons Attribution Non Commercial (CC BY-NC 4.0) license, which permits others to distribute, remix, adapt, build upon this work non-commercially, and license their derivative works on different terms, provided the original work is properly cited, appropriate credit is given, any changes made indicated, and the use is non-commercial. See: http://creativecommons.org/licenses/by-nc/4.0/.

ORCID iD

Manouk Admiraal http://orcid.org/0000-0002-2643-6246

\section{REFERENCES}

1 Weiser TG, Regenbogen SE, Thompson KD, et al. An estimation of the global volume of surgery: a modelling strategy based on available data. Lancet 2008;372:139-44.

2 Macrae WA. Chronic post-surgical pain: 10 years on. $\mathrm{Br} J$ Anaesth 2008;101:77-86.

3 Neuman MD, Bateman BT, Wunsch H. Inappropriate opioid prescription after surgery. Lancet 2019;393:1547-57.

4 Richebé P, Capdevila X, Rivat C. Persistent postsurgical pain: pathophysiology and preventative pharmacologic considerations. Anesthesiology 2018;129:590-607.

5 Tiippana E, Hamunen K, Heiskanen T, et al. New approach for treatment of prolonged postoperative pain: APS out-patient clinic. Scand J Pain 2016;12:19-24.

6 Clarke H, Azargive S, Montbriand J, et al. Opioid weaning and pain management in postsurgical patients at the Toronto General Hospital transitional pain service. Canadian Journal of Pain 2018;2:236-47.

7 Chan A-W, Tetzlaff JM, Altman DG, et al. Spirit 2013 statement: defining standard protocol items for clinical trials. Ann Intern Med 2013;158:200-7.

8 Chan A-W, Tetzlaff JM, Gøtzsche PC, et al. Spirit 2013 explanation and elaboration: guidance for protocols of clinical trials. BMJ 2013;346:e7586.

9 Hopewell S, Ravaud P, Baron G, et al. Effect of editors' implementation of consort guidelines on the reporting of Abstracts in high impact medical journals: interrupted time series analysis. BMJ 2012;344:e4178.

10 Calvert M, Kyte D, Mercieca-Bebber R, et al. Guidelines for inclusion of patient-reported outcomes in clinical trial protocols: the SPIRITPRO extension. JAMA 2018;319:483-94.

11 Schug SA, Lavand'homme P, Barke A, et al. The IASP classification of chronic pain for ICD-11: chronic postsurgical or posttraumatic pain. Pain 2019;160:45-52. 
12 Organization WH. ICD-11 2019, 2019. Available: https://icd.who.int aA

13 American Society of Anesthesiologists Task Force on Acute Pain Management. Practice guidelines for acute pain management in the perioperative setting: an updated report by the American Society of Anesthesiologists Task force on acute pain management. Anesthesiology 2012;116:248-73.

14 Watson JA, Ryan CG, Cooper L, et al. Pain neuroscience education for adults with chronic musculoskeletal pain: a mixed-methods systematic review and meta-analysis. J Pain 2019;20:1140.e1-1140. e22.

15 Stark PA, Myles PS, Burke JA. Development and psychometric evaluation of a postoperative quality of recovery score: the QoR-15. Anesthesiology 2013;118:1332-40.

16 Hamilton DF, Giesinger JM, Giesinger K. It is merely subjective opinion that patient-reported outcome measures are not objective tools. Bone Joint Res 2017;6:665-6.

17 Kleif J, Waage J, Christensen KB, et al. Systematic review of the QoR-15 score, a patient- reported outcome measure measuring quality of recovery after surgery and anaesthesia. $\mathrm{Br} J$ Anaesth 2018;120:28-36.

18 Shulman MA, Myles PS, Chan MTV, et al. Measurement of disabilityfree survival after surgery. Anesthesiology 2015;122:524-36.
19 TBustun NK, CHatterji S, et al. Measuring health and disability. manual for who disability Assesment schedule. WHODAS 2.0, 2010.

20 Shulman MA, Kasza J, Myles PS. Defining the minimal clinically important difference and Patient-acceptable symptom state score for disability assessment in surgical patients. Anesthesiology 2020;132:1362-70.

21 Rabin R, de Charro F. EQ-5D: a measure of health status from the EuroQol group. Ann Med 2001;33:337-43.

22 van Reenen BJ M, Stolk E, et al. EQ-5D-5L user guide. 3.0 ed, 2019.

23 M Versteegh M, M Vermeulen K, M A A Evers S, et al. Dutch tariff for the five-level version of EQ-5D. Value Health 2016;19:343-52.

24 Hays RD, Spritzer KL, Schalet BD, et al. PROMIS ${ }^{\circledR}-29$ v2.0 profile physical and mental health summary scores. Qual Life Res 2018;27:1885-91.

25 Myles PS, Myles DB, Galagher W, et al. Minimal clinically important difference for three quality of recovery scales. Anesthesiology 2016;125:39-45.

26 Sullivan GM, Artino AR. Analyzing and interpreting data from likerttype scales. J Grad Med Educ 2013;5:541-2.

27 Hochberg Y, Benjamini Y. More powerful procedures for multiple significance testing. Stat Med 1990;9:811-8. 